\title{
Image Feature Generation by Visio-Motor Map Learning towards Selective Attention
}

\author{
Takashi Minato and Minoru Asada \\ Dept. of Adaptive Machine Systems, Graduate School of Engineering, Osaka University \\ Suita, Osaka 565-0871, Japan \\ e-mail: minato@er.ams.eng.osaka-u.ac.jp, asada@ams.eng.osaka-u.ac.jp
}

\begin{abstract}
Visual attention is one of the key issues for robots to accomplish the given tasks, and the existing methods specify the image features and attention control scheme in advance according to the task and the robot. However, in order to cope with environmental changes and/or task variations, the robot should construct its own attention mechanism. As the first step towards selective attention, this paper presents a method for image feature generation by visio-motor map learning for a mobile robot. The teaching data construct the visio-motor mapping that constrains the image feature generation and state vector estimation as well. The resultant image feature and state vector are nothing but task-oriented. The method is applied to indoor navigation and soccer shooting tasks, and discussion is given.
\end{abstract}

\section{Introduction}

Biological systems have evolved their organs and acquired their own strategies so that they can survive in hostile environments through billions of years of evolution process. Visual attention can be regarded as a combination of such organs and strategies, that is, "vision" that brings a huge amount of data about the external world and "attention mechanism" that extracts the necessary and sufficient information from them for the system to achieve the mission at hand. Such a capability is desired in artificial systems too, and therefore, it has been one of the most typical but formidable issues in robotics and AI for long years.

Human beings can easily enjoy such a mechanism in various kinds of situations, and a number of researches focus on the early visual processing of human beings [1], improve the Treisman' model [2, 3], and apply Shanon's information of the observed image [4] in order to select the focus of attention in the view. The main issues of these works are the analysis of the human visual processing and the explanation for our attention mechanism.

Some of computer vision researchers focused on the view point selection (where to look) problem $[5,6]$ in order to disambiguate the descriptions for the observed image that is obtained by matching the image with the model database. The selection criterion is based on the statistics of the image data and actions (gaze control), if any, are intended to get the better observation for object recognition, but are not directly related to physical actions needed to accomplish a given task.

Self localization is the one of the issues in navigation task, and most of the works are based on a kind of geometric reconstruction from the observed image using a priori knowledge of the environment. Thrun [7] and Vlassis et al. [8] extracted the features correlated to the information of the self-localization of the mobile robot from the observed images based on the probabilistic method. Kröse and Bunschoten [9] decided the robot direction, i.e., camera direction by minimizing the conditional entropy of the robot position given the observations.

The existing approaches mentioned above mostly specify the kinds of image features in advance and adopt a sort of attention mechanism based on the designers intuition having considered the given task. However, in order to cope with environmental changes and/or task variations, the robot should generate image features and construct its own attention mechanism. As the first step towards selective attention, this paper presents a method for image feature generation by visio-motor map learning for a mobile robot. The teaching data construct the visio-motor mapping that constrains the image feature generation and state vector estimation for the action selection as well. The resultant image feature and state vector could be nothing but they are task-oriented. The method is applied 


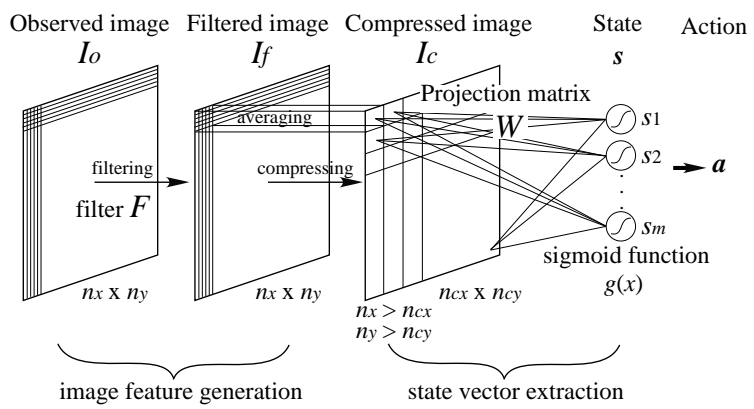

Figure 1: Image feature generation and action selection model

to indoor navigation and soccer shooting tasks.

In the existing approaches, there have been some methods to construct the visual state spaces through the task executions (e.g.[10, 11]). These methods can construct the task-oriented state vector, but they don't focus on the image features. Our proposed method constructs the task-oriented visual state space and image feature which is useful for the selective attention.

The rest of the paper is organized as follows. First, the basic idea for image feature generation is described along with the learning formulation. Here, the projection matrix from the extracted image feature to the state vector is introduced to consequently determine the optimal action. Next, the experimental results are given to show the validity of the proposed method. Finally, discussion on the attention mechanism suggested from the current results is given towards the next step.

\section{Image feature generation}

\section{$2.1 \quad$ A basic idea}

Fig.1 shows the proposed model of the system for image feature generation and action selection. The reason why adopting two stage learning is that we expect the former more general and less task specific while the latter vice versa. In other words, at the image feature generation stage, the interactions between raw data are limited inside local area while the connections between the image features and the states spread over the entire space to represent more global interactions. A similar structure can be found in the synapse connections in our brain, where the retinal signals geometrically close to each other are mapped to nearby regions in the early visual processing area while the post-processing and therefore more abstracted information is spread out the whole brain via a number of synapse connections (ex. [12]).

We prepare the image filter $F$ to generate the image features. The robot estimates its state $s$ from the filtered image $I_{f}$ and decides the action appropriate to the current state $\boldsymbol{s}$. In order to avoid curse of dimension, we compress the filtered image $I_{f}$ into $I_{c}$ from which the state vector is extracted by a projection matrix $W$. We can regard $W$ as a kind of attention mechanism because it connects the filtered image $I_{c}$ to the state space, that is, it tells which part in the view is more important to estimate each state, and finally to decide the optimal action. Therefore, the problem is how to learn $F$ and $W$.

In order to reflect the task constraints, we use the supervised successful instances (a training set). $F$ and $W$ are computed by minimizing the conditional entropy of the action given the state on the training set.

In this paper we prepare a $3 \times 3$ spatial filter $F_{s}$ and a color filter $F_{c}$ as follows:

- a $3 \times 3$ spatial filter $F_{s} \in \Re^{3 \times 3}$ :

$$
\begin{aligned}
& \bar{I}_{x y}=f_{s 11} I_{x-1 y-1}+f_{s 12} I_{x y-1}+f_{s 13} I_{x+1 y-1} \\
& +f_{s 21} I_{x-1 y}+f_{s 22} I_{x y}+f_{s 23} I_{x+1 y} \\
& +f_{s 31} I_{x-1 y+1}+f_{s 32} I_{x y+1}+f_{s 33} I_{x+1 y+1} \text {, } \\
& I_{f x y}=g\left(\bar{I}_{x y}\right) .
\end{aligned}
$$

- a color filter $F_{c} \in \Re^{3}$ :

$$
\begin{gathered}
\bar{I}_{x y}=f_{c 1} I_{r x y}+f_{c 2} I_{g x y}+f_{c 3} I_{b x y}, \\
I_{f x y}=g\left(\bar{I}_{x y}\right),
\end{gathered}
$$

where $x$ and $y$ denote the position of the pixel, $I, I_{r}, I_{g}$ and $I_{b}$ the gray, red, green and blue components of the observed image, respectively, and $g(\cdot)$ a sigmoid function. For example, the following $F_{s}$ and $F_{c}$ represent a vertical edge filter and a brightness one, respectively.

$$
\begin{gathered}
F_{s}=\left(\begin{array}{ccc}
-1 & 0 & 1 \\
-1 & 0 & 1 \\
-1 & 0 & 1
\end{array}\right) \\
F_{c}=\left(\begin{array}{lll}
0.2990 & 0.5870 & 0.1140
\end{array}\right)^{T} .
\end{gathered}
$$

\subsection{Learning method}

In the teaching stage the robot collect the $i$-th pair

$$
T_{i}=<I_{o i}, \boldsymbol{a}_{i}>,
$$


where $I_{O}$ is the observed image, $\boldsymbol{a} \in \Re^{l}$ is the supervised robot action executed after the robot observes $I_{o}$ and $i$ denotes the data number. In the case of a mobile robot, $l$ is two.

The state of the robot $s \in \Re^{m}$ is extracted by $W \in$ $\Re^{m \times n_{c x} n_{c y}}$. Let $\boldsymbol{i}_{c} \in \Re^{n_{c x} n_{c y}}$ be the one dimensional representation of $I_{c}$, then

$$
\boldsymbol{s}=\boldsymbol{g}\left(W \boldsymbol{i}_{c}\right),
$$

where $\boldsymbol{g}(\cdot)$ is a vector function of which components are sigmoid functions.

To evaluate $F$ and $W$, we use the conditional entropy $H(\boldsymbol{a} \mid \boldsymbol{s})$ :

$$
H(\boldsymbol{a} \mid \boldsymbol{s})=-\int p(\boldsymbol{s}) \int p(\boldsymbol{a} \mid \boldsymbol{s}) \log p(\boldsymbol{a} \mid \boldsymbol{s}) d \boldsymbol{a} d \boldsymbol{s},
$$

where $p(\cdot)$ denotes the probabilistic density. To approximate $H(\boldsymbol{a} \mid \boldsymbol{s})$, we use the risk function $R$ [8].

$$
\begin{aligned}
R & =-\frac{1}{N} \sum_{d}^{N} \log p\left(\boldsymbol{a}_{d} \mid \boldsymbol{s}_{d}\right) \\
& =-\frac{1}{N} \sum_{d}^{N} \log \frac{p\left(\boldsymbol{a}_{d}, \boldsymbol{s}_{d}\right)}{p\left(\boldsymbol{s}_{d}\right)}
\end{aligned}
$$

where $N$ is the size of the teaching data set. To model $p(\boldsymbol{a}, \boldsymbol{s})$ and $p(\boldsymbol{s})$, we use the kernel smoothing [13].

$$
\begin{gathered}
p(\boldsymbol{s})=\frac{1}{N} \sum_{q}^{N} K_{s}\left(\boldsymbol{s}, \boldsymbol{s}_{q}\right) \\
p(\boldsymbol{a}, \boldsymbol{s})=\frac{1}{N} \sum_{q}^{N} K_{a}\left(\boldsymbol{a}, \boldsymbol{a}_{q}\right) K_{s}\left(\boldsymbol{s}, \boldsymbol{s}_{q}\right)
\end{gathered}
$$

where

$$
\begin{aligned}
& K_{s}\left(\boldsymbol{s}, \boldsymbol{s}_{q}\right)=\frac{1}{(2 \pi)^{m / 2} h_{s}^{m}} \exp \left(-\frac{\left\|\boldsymbol{s}-\boldsymbol{s}_{q}\right\|^{2}}{2 h_{s}^{2}}\right), \\
& K_{a}\left(\boldsymbol{a}, \boldsymbol{a}_{q}\right)=\frac{1}{(2 \pi)^{l / 2} h_{a}^{l}} \exp \left(-\frac{\left\|\boldsymbol{a}-\boldsymbol{a}_{q}\right\|^{2}}{2 h_{a}^{2}}\right),
\end{aligned}
$$

$h_{s}$ and $h_{a}$ are the width of the kernels. $R$ can be regarded as the Kullback-Leibler distance between $p\left(\boldsymbol{a} \mid \boldsymbol{s}_{d}\right)$ and a unimodal density sharply peaked at $\boldsymbol{a}=\boldsymbol{a}_{d}$. By minimizing $R$, we can bring $p(\boldsymbol{a} \mid \boldsymbol{s})$ close to the unimodal density, that is, the robot can uniquely decide the action $\boldsymbol{a}$ from the state $\boldsymbol{s}$.

Using the steepest gradient method, we obtain a pair of $F$ and $W$ which minimize $R$ :

$$
F \leftarrow F-\alpha_{f} \frac{\partial R}{\partial F}, W \leftarrow W-\alpha_{w} \frac{\partial R}{\partial W},
$$

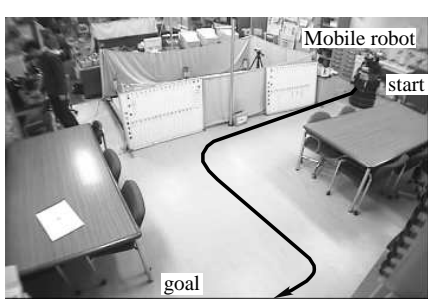

(a) Task 1

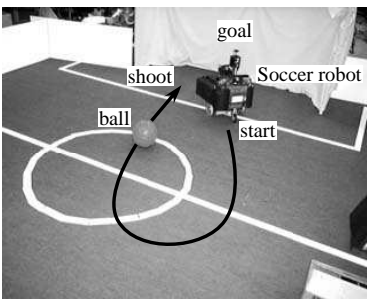

(b) Task 2
Figure 2: Task

where $\alpha_{f}$ and $\alpha_{w}$ are the step size parameters.

After learning the robot executes the action $\boldsymbol{a}$ derived from its state $s$ computed from the observed image as follows:

$$
\boldsymbol{a}=\arg \max _{\boldsymbol{a}^{\prime}} p\left(\boldsymbol{a}^{\prime} \mid \boldsymbol{s}\right) .
$$

To find the maximum value, we adopt the coarse-fine search strategy.

\section{$3 \quad$ Experiments}

\subsection{Task and assumptions}

We applied the proposed method to the indoor navigation task with the Nomad mobile robot (Fig.2(a)) and the shooting ball task of the soccer robot (Fig.2(b)). The mobile robot shown in Fig.2(a) is equipped with stereo cameras and we use only the left camera image. The soccer robot shown in Fig.2(b) is equipped with a single camera directed ahead. The size of observed image is $64 \times 54$ and the values of $I, I_{r}, I_{g}$ and $I_{b}$ are normalized to $\left[\begin{array}{ll}0 & 1\end{array}\right]$. The robots can execute translational speed $v$ and steering speed $\omega$ independently, so the action vector is represented as

$$
\boldsymbol{a}=(v, \omega)^{T},
$$

where $v$ and $\omega$ are normalized to [-1 1$]$, respectively. We define the size of the compressed filtered image as $8 \times 6$ and the dimension of state as $m=2$. The sigmoid function $g$ is

$$
g(x)=\frac{1}{1+\exp \left(-\frac{x-\theta}{c}\right)},
$$

where $\theta=0.0$ and $c=0.2$. 


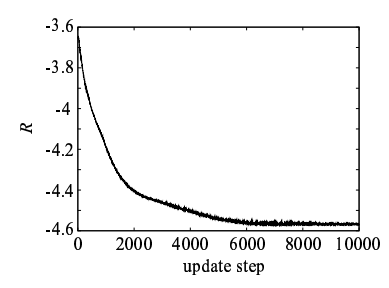

(a) Model with $F_{s}$

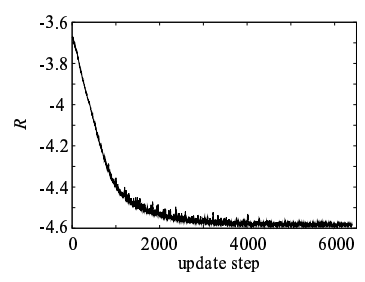

(b) Model with $F_{c}$
Figure 3: Learning curves of $R$

\subsection{Learning results}

At the teaching stage, we gave 158 pairs of images and actions in the task 1 , and 100 pairs in the task 2 . In each task we tested the two models (Fig.1) with a spatial filter $F_{s}$ and a color filter $F_{c}$, separately. We initialized the components of $W$ by random small number and

$$
\begin{gathered}
F_{s}=\left(\begin{array}{lll}
0.1 & 0.1 & 0.1 \\
0.1 & 0.1 & 0.1 \\
0.1 & 0.1 & 0.1
\end{array}\right) \quad \text { (smoothing), } \\
F_{c}=\left(\begin{array}{lll}
0.2990 & 0.5870 & 0.1140
\end{array}\right)^{T} \text { (brightness). }
\end{gathered}
$$

\subsubsection{Task 1: simple navigation}

Fig. 3 shows the changes of $R$ in the case of $F_{s}$ and $F_{c}$ models. $F$ and $W$ are learned so as to decrease $R$. Fig. 4 shows the distributions of the state on the teaching data set in the case of the model with $F_{s}$. To show the relation between the states and actions, we labeled the action indices as follows:

- $v \geq 0.6$ : forward,

- $v \leq-0.6$ : backward,

- $-0.6<v<0.6$ and $\omega<0.0$ : right turn, and

- $-0.6<v<0.6$ and $\omega>0.0$ : left turn.

As we can see from these figures, the state space can be roughly classified in terms of the kind of action. That is, the state space is constructed to correlated to the action space. However it seems difficult to reveal a physical interpretation from this relationship.

The generated $F_{s}$ and $F_{c}$ are shown below:

$$
F_{s}=\left(\begin{array}{rrr}
-0.8915 & -0.5995 & -0.06528 \\
-0.9696 & -0.4790 & 1.357 \\
-0.2482 & 0.1021 & 2.756
\end{array}\right)
$$

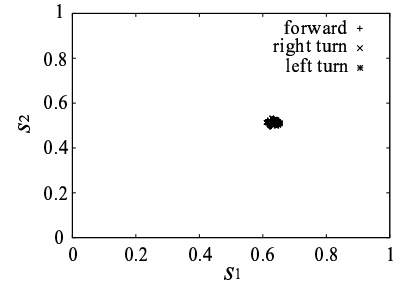

(a) Initial state space

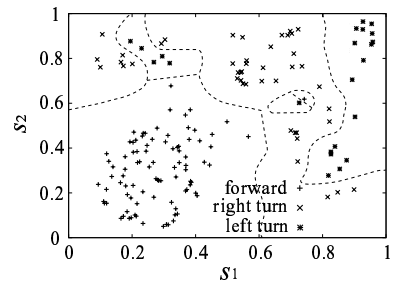

(b) Learned state space
Figure 4: State distributions

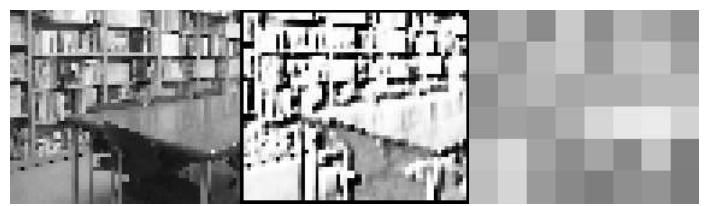

(a) $I o$ (b) If

(c) $I c$
Figure 5: Example of the filtered image with $F_{s}$

$$
F_{c}=\left(\begin{array}{lll}
-0.4233 & 1.464 & -0.1718
\end{array}\right)^{T} .
$$

Figs. 5 and 6 show the examples of the filtered images. As we can see from Fig. $5, F_{s}$ shows the characteristic to extract vertical and horizontal edges. However $F_{c}$ does not show remarkable characteristic because there are not salient color objects in the environment (our laboratory). Intuitively, it implies that the generated $F_{s}$ is good at a navigation task of a mobile robot.

\subsubsection{Task 2: shooting a ball}

The generated $F_{s}$ and $F_{c}$ are shown below. The examples of the filtered image are shown in Figs.7 and 8.

$$
\begin{gathered}
F_{s}=\left(\begin{array}{rrr}
-3.384 & -1.953 & -1.686 \\
0.3491 & -1.350 & 0.5363 \\
1.656 & -1.208 & 5.223
\end{array}\right), \\
F_{c}=\left(\begin{array}{lll}
1.836 & 1.616 & -4.569
\end{array}\right)^{T} .
\end{gathered}
$$

$F_{s}$ shows the characteristic to extract horizontal edges (see Fig.7). $F_{c}$ emphasizes the red ball and yellow goal but inhibits the white line and wall. This is equivalent to a characteristic of a reversed U component of YUV image. The generated $F_{c}$ is good at a soccer robot task in the colored soccer field. 


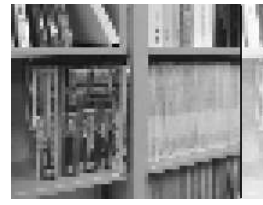

(a) $I o$

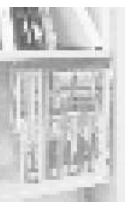

(b) If

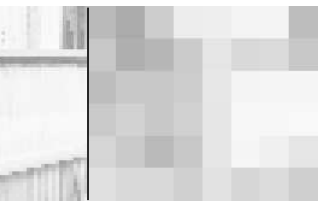

(c) $I c$
Figure 6: Example of the filtered image with $F_{c}$

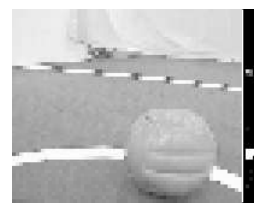

(a) Io

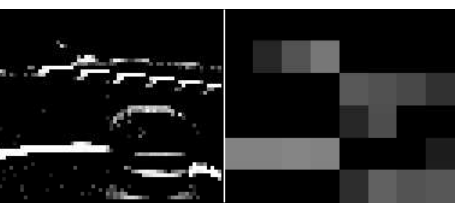

(b) If

(c) $I_{c}$
Figure 7: Example of the filtered image with $F_{s}$

\subsection{Learned behavior}

To verify the validity of the learned model, we applied the model with $F_{s}$ (task 1 ) to a navigation task of the Nomad mobile robot (see Fig.2(a)). Fig.9 shows a sequence of the acquired behavior. The estimated states in this experiment are not exactly coincident with the states computed from the teaching data set, but the robot accomplished the task. Hence it implies that this model is an effective representation for the task and environment.

\section{Discussion and future work}

In this paper we proposed the method to generate an image feature and to learn a projection matrix from the image feature to the state, that suggests which part in the view is important, that is, a gaze selection by visio-motor mapping. The generated image features are appropriate for the task and environment. Also the acquired projection matrices give appropriate gaze selection for the task and environment. To show this, we illustrate the absolute value of $W$ acquired in the model with $F_{c}$ in the task 2. Figs.10(a) and (b) show the values of components of $W$ related to the first and second components of the state $s$, respectively. In these figures brighter pixels are more closely related to the state vector, that is, the robot gazes this parts in the view. Therefore we can regard that a projection matrix gives a gaze selection.

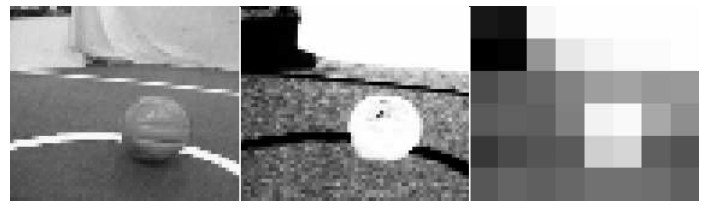

(a) Io

(b) If

(c) $I c$

Figure 8: Example of the filtered image with $F_{c}$

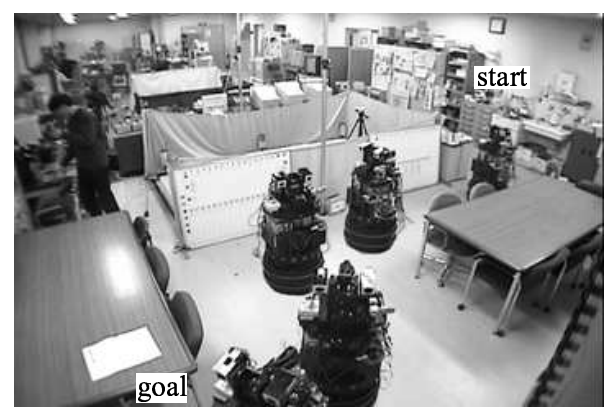

Figure 9: Acquired behavior

In this paper we defined the dimension of the state vector as two heuristically. However, as a result, this number was appropriate. Fig.11(a) shows the relationship between the number of dimension and $R$ in the case of learning the model with $F_{s}$ in the task 1 , and Fig.11(b) shows the relationship between the number of dimension and the action estimation error. This error shows the sum of error norms between the estimated action and the supervised action. From these figures we can see that the necessary number of dimension is two to estimate the action from the state.

Next, in order to show the effectiveness of the method we compare the acquired model with an attention control scheme specified by the designer heuristically. For example we specify the following scheme:

- Image feature is disparity.

- We extract the minimum disparity region (the region furthermost from the robot) in the disparity image and define the center of the region as the state $s$ (two dimensions).

Using the same teaching data set as that of task 1, we compute ${ }^{\dagger}$ the state distribution similar to Fig.4.

\footnotetext{
$\dagger$ At the training stage of the experiment in section 3 , we also have collected the right camera images, so we can compute the disparity images.
} 

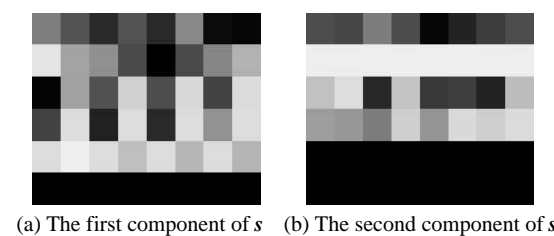

Figure 10: Projection matrix $W$

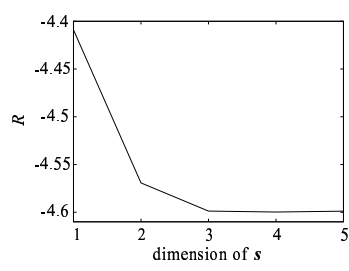

(a) $R$

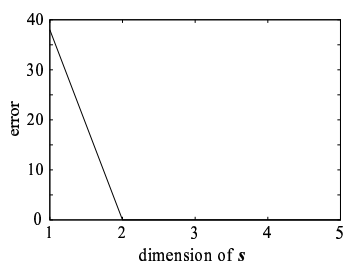

(b) Estimation error
Figure 11: Effect of the dimension of the state vector

The result is shown in Fig.12. Comparing Fig.4(b) and Fig.12, we can see that the states do not appropriately correlated to the action in Fig.12. In Fig.12 $R$ is -4.211 larger than that of Fig. $4(\mathrm{~b})(R=-4.569)$. This fact shows that the proposed method can learn effective image feature and gaze selection for the task.

In the sequence of Fig.9 there are some cases that the robot decides the actions with relatively low probability $p(\boldsymbol{a} \mid \boldsymbol{s})$, that is, the robot is not so sure about its action decision. Therefore, it seems necessary for the robot to select multiple image features from the image feature set to accomplish more complicated tasks. Now, we are investigating how to integrate the proposed method and the image feature selection method based on the information theoretic criterion [14].

\section{References}

[1] A. Treisman and A. Gelade. A feature integration theory of attention. Cognitive Psychology, Vol. 12, pp. 97-136, 1980.

[2] J. M. Wolfe, K. R. Cave, and S. L. Franzel. Guided search: An alternative to the feature integration model. Journal of Experimental Psychology: Human Perception and Performance, Vol. 15, No. 3, pp. 419433, 1989.

[3] P. Laar and S. Gielen. Task-dependent learning of attention. Neural Networks, Vol. 10, No. 6, pp. 981992, 1997.

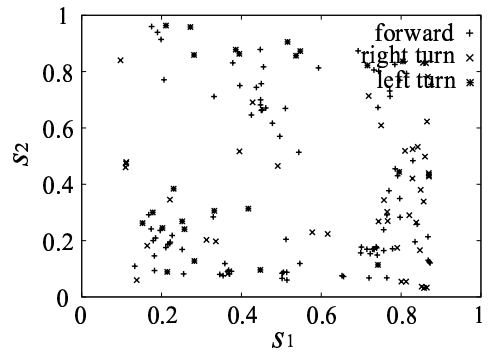

Figure 12: Distribution of the states defined by the designer

[4] Y. Takeuchi, N. Ohnishi, and N. Sugie. Active vision system based on information theory. Systems and Computers in Japan, Vol. 29, No. 11, pp. 31-39, 1998.

[5] S. K. Nayar, H. Murase, and S. A. Nene. Parametric Appearance Representation in Early Visual Learning, chapter 6. Oxford University Press, 1996.

[6] T. Arbel and F. P. Ferrie. Viewpoint selection by navigation through entropy maps. In Proceedings of the Seventh International Conference on Computer Vision, pp. 248-254, 1999.

[7] S. Thrun. Bayesian landmark learning for mobile robot localization. Machine Learning, Vol. 31, No. 1, 1998.

[8] N. Vlassis, R. Bunschoten, and B. Kröse. Learning task-relevant features from robot data. In Proceedings of the 2001 IEEE International Conference on Robotics and Automation, pp. 499-504, 2001.

[9] B. J. A. Kröse and R. Bunschoten. Probabilistic localization by appearance models and active vision. In Proceedings of the 1999 IEEE International Conference on Robotics and Automation, pp. 2255-2260, 1999.

[10] T. Nakamura. Development of self-learning visionbased mobile robots for acquiring soccer robots behaviors. In Proceedings of the 1998 IEEE International Conference on Robotics and Automation, pp. 2592-2598, 1998.

[11] H. Ishiguro, M. Kamiharako, and T. Ishida. State space construction by attention control. In Proceedings of the Sixteenth International Joint Conference on Artificial Intelligence, pp. 1131-1137, 1999.

[12] D. J. Fellman and D. C. Van Essen. Distributed hierarchical processing in the primate cerebral cortex. Cerebral Cortex, Vol. 1, pp. 1-47, 1991.

[13] M. P. Wand and M. C. Jones. Kernel Smoothing. Chapman \& Hall, 1995.

[14] T. Minato and M. Asada. Selective attention mechanism for mobile robot based on information theory. In Proceedings of the 18th Annual Conference of the Robot Society of Japan, pp. 811-812, 2000. (in Japanese). 